BULL. AUSTRAL. MATH. SOC.

VOL. $13(1975), 375-387$.

\title{
Relatively flat modules
}

\section{Paul E. Bland}

If $\left(A^{\prime}, B^{\prime}\right),\left(B^{\prime}, C^{\prime}\right)$ and $(A, B),(B, C)$ are torsion-torsion free theories on $R^{M}$ and $M_{R}$ respectively which are generated by an idempotent ideal $I$ of $R$, then $M \in R^{M}$ is said to be relatively flat if $(\cdot) \otimes{ }_{R}{ }^{M}$ preserves short exact sequences $0 \rightarrow L+X \rightarrow N \rightarrow 0$ in $M_{R}$ with $N \in B$. Several characterizations of relatively flat modules are given and it is shown that any module $M \in{ }_{R}^{M}$ which is codivisible with respect to $\left(A^{\prime}, B^{\prime}\right)$ is relatively flat. In addition, when ( $\left.A^{\prime}, B^{\prime}\right)$ is hereditary, it is proven that $M \in{ }_{R} M$ is relatively flat if and only if $M / I M$ is a flat $R / I$-module. Finally, a relatively flat dimension for $M \in R^{M}$ and a left global relatively flat dimension for $R$ are defined and it is shown, again when $\left(A^{\prime}, B^{\prime}\right)$ is hereditary, that the left global relatively flat dimension of $R$ coincides with the left global flat dimension of $R / I$.

\section{Introduction}

Throughout this paper $R$ will denote an associative ring with identity and our attention will be confined to the categories $R^{M}$ and $M_{R}$ of unital left and right $R$-modules respectively. Our purpose is to study relatively flat modules in the setting of torsion-torsion free theories which are generated by an idempotent ideal of $R$. The reader is referred to [2], [5], and [7] for the general results and terminology on torsion theories.

Received 5 September 1975. 
If $(A, B)$ is a torsion theory on $M_{R}$, then a right $R$-module $M$ is said to be divisible (codivisible) if given an exact sequence $0 \rightarrow L \rightarrow X \rightarrow N \rightarrow 0$ in $M_{R}$, where $N$ is torsion ( $L$ is torsion free), the induced map $\operatorname{hom}_{R}(X, M) \rightarrow \operatorname{hom}_{R}(L, M) \quad\left(\operatorname{hom}_{R}(M, X) \rightarrow \operatorname{hom}_{R}(M, N)\right)$ is an epimorphism. By taking $X$ to be projective (injective), we see that $M$ is divisible (codivisible) if and only if $\operatorname{ext}_{R}^{1}(N, M)=0$ for every torsion module $N\left(\operatorname{ext}_{R}^{1}(M, L)=0\right.$ for every torsion free module $\left.L\right)$. Divisible modures are due to Lambek [5] and codivisible modules were introduced in [1].

In [4], Jans calls a class $B$ of modules in $M_{R}$ a torsion-torsion free class if $B$ is closed under taking submodules, factors, extensions, direct products and direct sums. By saying that $B$ is closed under extensions we mean that $M \in B$ whenever there is a submodule $N$ of $M$ such that $N$ and $M / N$ are in $B$. For such a torsion-torsion free class $B$ there exist classes $A$ and $C$ of modules such that $(A, B)$ and $(B, C)$ are torsion theories on $M_{R}$. We shall refer to such a pair $(A, B),(B, C)$ as a torsion-torsion free theory on $M_{R}$. Jans has also shown [4, Corollary 2.2] that there is a one-to-one correspondence $I \leftrightarrow B=\{M \mid M I=0\}$ between idempotent ideals $I$ of $R$ and torsiontorsion free classes $B$ in $M_{R}$. Thus it follows that:

(I) $A=\{M \mid M I=M\}=\left\{M \mid M \otimes \otimes_{R} \cong M\right\}$;

(2) $B=\{M \mid M I=0\}=\left\{M \mid M \otimes{ }_{R} I=0\right\}$;

(3) $C=\left\{M \mid \operatorname{hom}_{R}(B, M)=0\right.$ for all $\left.B \in B\right\}$;

(4) $\mathrm{A}(M)=M I$ for any $M \in M_{R}$ where $A(M)$ denotes the torsion submodule of $M$ with respect to $(A, B)$;

(5) the idempotent filter of right ideals of $R$ associated with $(B, C)$ is given by

$$
F(R)=\{K \mid K \supseteq I, K \text { a right ideal of } R\} \text {. }
$$

Obviously, a given idempotent ideal $I$ of $R$ generates a torsion-torsion 
free theory $\left(A^{\prime}, E^{\prime}\right),\left(B^{\prime}, C^{\prime}\right)$ on $R^{M}$ and a torsion-torsion free theory $(A, B),(B, C)$ on $M_{R}$. Notice that $A^{\prime}(R)=A(R)=I$. Throughout the remainder of this paper we will suppose, unless stated otherwise, that $I$ is an idempotent ideal of $R$ and that $\left(A^{\prime}, B^{\prime}\right),\left(B^{\prime}, C^{\prime}\right)$ and $(A, B),(B, C)$ are as above. For $M \in R_{R}^{M}$ or $M_{R}, M^{*}=h_{Z}(M, Q / Z)$ will denote the character module of $M$. Before beginning we record the fact that many of the following definitions and theorems reduce to definitions and theorems of "classical" homological algebra when $I=0$.

\section{E-flat modules}

If $E$ is the class of all short exact sequences $0 \rightarrow L \rightarrow X \rightarrow N \rightarrow 0$ in $M_{R}$ with $N \in B$, then $M \in R_{R}^{M}$ is said to be flat relative to $E$ or simply E-flat if $(\cdot) \otimes R^{M}$ preserves short exact sequences in $E$. It is easy to see that $M$ is E-flat if and only if $\operatorname{tor}_{1}^{R}(N, M)=0$ for all $N \in B$.

THEOREM 2.1. The following are equivalent for any $M \in{ }_{R} M$ :

(1) $M$ is E-flat;

(2) $M^{*}$ is divisible with respect to $(B, C)$;

(3) $K \otimes{ }_{R^{M}} \cong K M$ canonically for each $K \in F(R)$;

(4) if $M \cong E / N$ where $E$ is E-flat, then $K E \cap N=K N$ for each $K \in F(R)$;

(5) $\operatorname{tor}_{1}^{R}(R / K, M)=0$ for all $K \in F(R)$.

Proof. We will show (I) $\Rightarrow(5) \Rightarrow(3) \Rightarrow(2) \Rightarrow(1)$ and then (1) $\Leftrightarrow(4)$.

(1) $\Rightarrow(5)$ is obvious since if $K \in F(R)$, then $R / K \in B$.

(5) $\Rightarrow(3)$. If $K \in F(R)$, then $0 \rightarrow K \stackrel{j}{\rightarrow} R \rightarrow R / K \rightarrow 0$ is in $E$ and so $0=\operatorname{tor}_{1}^{R}(R / K, M) \rightarrow K \otimes R^{M} \stackrel{I \otimes j}{\longrightarrow} R \otimes R^{M}$ is exact. Thus if $\varphi: R \otimes R^{M} \rightarrow M$ is the canonical isomorphism, then $K \otimes R^{M} \cong \varphi \circ(1 \otimes j)\left(K \otimes R_{R}^{M}\right)=K M$. 
(3) $\Rightarrow$ (2). If $K \otimes R^{M} \cong K M$ canonically for each $K \in F(R)$, then $K \otimes R^{M} \rightarrow M: \sum_{i=1}^{n} k_{i} \otimes m_{i} \rightarrow \sum_{i=1}^{n} k_{i} m_{i}$ is a monomorphism. Hence we have an epimorphism $\operatorname{hom}_{R}\left(R, M^{*}\right) \rightarrow \operatorname{hom}_{R}\left(K, M^{*}\right)$ since $\left(K \otimes R^{M}\right)^{*} \cong \operatorname{hom}_{R}\left(K, M^{*}\right)$ and $M^{*} \cong \operatorname{hom}_{R}\left(R, M^{*}\right)$. Thus, it follows from the Generalized Baer's Criterion [3, Proposition 3.2] that $M^{*}$ is divisible with respect to $(B, C)$.

(2) $\Rightarrow$ (1). If $0 \rightarrow L \rightarrow X \rightarrow N \rightarrow 0$ is in $E$, then

$$
0 \rightarrow \operatorname{hom}_{R}\left(N, M^{*}\right) \rightarrow \operatorname{hom}_{R}\left(X, M^{*}\right) \rightarrow \operatorname{hom}_{R}\left(L, M^{*}\right) \rightarrow 0
$$

is exact. Thus $0 \rightarrow\left(N \otimes R^{M}\right)^{*} \rightarrow\left(X \otimes R^{M}\right)^{*} \rightarrow\left(L \otimes R^{M}\right)^{*} \rightarrow 0$ is exact and so $0 \rightarrow L \otimes R^{M} \rightarrow X \otimes R^{M} \rightarrow N \otimes R^{M} \rightarrow 0 \quad$ is exact.

Finally, let us show that (1) $\Leftrightarrow(4)$.

$(1) \Rightarrow(4)$. For each $K \in(R)$, the exact sequence $N \stackrel{j}{\longrightarrow} E \stackrel{\varphi}{\longrightarrow} M \rightarrow 0$ where $N=\operatorname{ker} \varphi$ and $j$ is the canonical injection yields a commutative diagram

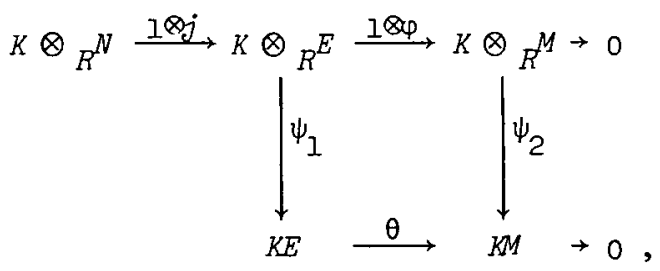

where $\psi_{1}$ and $\psi_{2}$ are the canonical isomorphisms given by (3) and $\theta: K E \rightarrow K M: \sum_{i=1}^{n} k_{i} x_{i} \rightarrow \sum_{i=1}^{n} k_{i} \varphi\left(x_{i}\right)$. Hence $K N=\psi_{1} \circ(1 \otimes j)\left(K \otimes R^{N}\right)=\psi_{1}(\operatorname{ker}(1 \otimes \varphi))=\operatorname{ker} \theta=K E \cap N$.

$(4) \Rightarrow(1)$. The exact sequence $N \stackrel{j}{\rightarrow} E \stackrel{\varphi}{\rightarrow} M \rightarrow 0$ yields, for each $K \in F(R)$, a diagram 


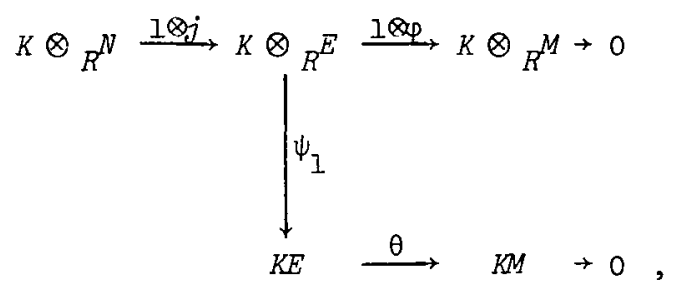

where $\psi_{1}$ and $\theta$ are as above. Since $K N=K E \cap N, K E / K N \cong K M$.

Notice also that since $\psi_{1} \circ(1 \otimes j)\left(K \otimes{ }_{R} N\right)=K N, \psi_{1}$ induces an isomorphism $\frac{K \otimes_{R} E}{(1 \otimes j)\left(K \otimes_{R} N\right)} \cong \frac{K E}{K N}$. But $\frac{K \otimes_{R} E}{(1 \otimes j)\left(K \otimes_{R} N\right)}=\frac{K \otimes_{R} E}{\operatorname{ker}\left(1 \otimes x_{p}\right)} \cong K \otimes{ }_{R}^{M}$. Hence we have an isomorphism $\psi_{2}: K \otimes R^{M} \rightarrow K M$ which can easily be shown to be the canonical isomorphism. Thus (3) holds and so $M$ is E-flat.

In passing we note that if $\left\{_{a}\right\} \quad(a \in I)$ is a family of modules in $R^{M}$, then $\oplus M_{a}(a \in I)$ is E-flat if and only each $M_{a}$ is E-flat. We now need the following

LEMMA 2.2. If $M \in M_{R}$, then $M \in B$ if and only if $M^{*} \in B^{\prime}$.

Proof. If $M \in B$, then $M I=0$ and so if $x f \in I M^{*}$, then $(x f)(m)=f(m x)=f(0)=0$ for each $m \in M$. Thus $x f=0$ and consequently, $I M^{*}=0$. Therefore $M^{*} \in B^{\prime}$. Conversely, suppose that $M^{*} \in B^{\prime}$; then $\left(M^{*}\right)^{*}=M^{* *} \in B$. But $M$ embeds in $M^{* *}$ and so $M \in B$ since $B$ is closed under submodules and isomorphic images.

In the proof of the following theorem if $f: M \rightarrow N$ is an $R$-homomorphism, then $f^{*}$ will denote the $R$-homomorphism $N^{*} \rightarrow M^{*}: g \rightarrow g \circ f$.

THEOREM 2.3. If $M \in{ }_{R}^{M}$ is codivisible with respect to ( $\left.A^{\prime}, B^{\prime}\right)$, then $M$ is E-flat.

Proof. Let $0 \rightarrow L \stackrel{j}{\longrightarrow} X \stackrel{k}{\longrightarrow} N \rightarrow 0$ be in $E$ and suppose that $f: L \rightarrow M^{*}$ is $R$-linear. By taking character modules we obtain a diagram 


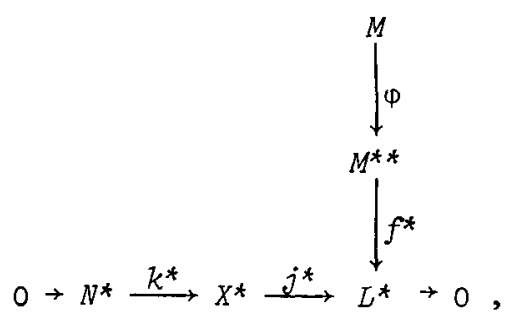

where $\varphi$ is the canonical embedding $M \rightarrow M^{* *}: m \rightarrow \varphi_{m}$ and $\varphi_{m}: M^{*} \rightarrow Q / Z: h \rightarrow h(m)$. Since $N^{*} \in B^{\prime}$ (Lemma 2.2) and $M$ is codivisible with respect to $\left(A^{\prime}, B^{\prime}\right)$ this diagram can be completed commutatively by an $R$-homomorphism $g: M \rightarrow X^{*}$. Thus if $u, v$, and $w$ are the canonical embeddings shown below, then we have a commutative diagram

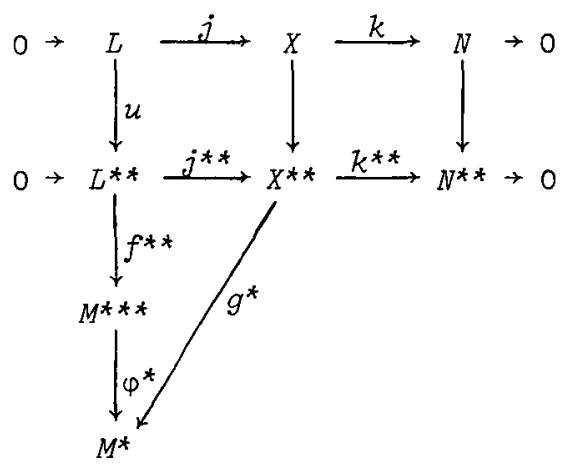

But if $x \in L$, then

$$
\left(\varphi^{*} \circ f^{* *} \circ u\right)(x)=\varphi^{*}\left(f^{* *}\left(u_{x}\right)\right)=\varphi^{*}\left(u_{x} \circ f^{*}\right)=u_{x} \circ f^{*} \circ \varphi,
$$

and so for $m \in M$,

$$
\left(u_{x} \circ f^{*} \circ \varphi\right)(m)=u_{x}\left(\varphi_{m} \circ f\right)=\left(\varphi_{m} \circ f\right)(x)=f(x)(m) .
$$

Thus $\varphi^{*} \circ f^{* *} \circ u=f$ and therefore we have shown that $M^{*}$ is divisible with respect to $(B, \mathcal{C})$. Hence, by Theorem $2.1, M$ is $E$-flat.

The following theorem relates $E$-flat modules to flat $R / I$-modules.

THEOREM 2.4. If $\left(A^{\prime}, B^{\prime}\right)$ is hereditary, then $M \in{ }_{R}^{M}$ is E-flat if and only if $M / M$ is a flat R/I-module.

Proof. Suppose that $M$ is E-flat and let $\varphi: C \rightarrow M$ be an 
$R$-epimorphism where $C$ is codivisible with respect to $\left(A^{\prime}, B^{\prime}\right)$. If $\bar{\varphi}: C / I C \rightarrow M / I M: x+I C \rightarrow \varphi(x)+I M$ is the induced $R / I$-epimorphism and $N=\operatorname{ker} \varphi$, then ker $\bar{\varphi}=(N+I C) / I C$. Hence, since $C / I C$ is a projective $R / I$-module [6, Theorem 8], to show that $M / I M$ is a flat $R / I$-module it suffices to show that $(N+I C) / I C \cap K C / I C \subseteq(K N+I C) / I C$ for any $K \in F(R)$. Let $x=n+I C \in(N+I C) / I C, n \in N$, and $x=m+I C \in K C / I C, m \in K C$; then $n-m \in I C \subseteq K C$ and so $n \in K C$. But, by Theorem 2.3; $C$ is E-flat and therefore, by Theorem 2.1, $K C \cap N=K N$. Hence $n \in K N$ and therefore $x \in(K N+I C) / I C$.

Conversely, suppose that $M / I M$ is a flat $R / I$-module and let $\varphi: C \rightarrow M$ and $N$ be as above. Let $K \in F(R)$ and suppose that $x \in K C \cap N$. Since $x \in K C$ and $x \in N$,

$$
x+I C \in(N+I C) / I C \cap K C / I C=(K N+I C) / I C .
$$

If $x+I C=y+I C, y \in K N \subseteq N$, then $x-y \in I C \cap N$. But $\left(A^{\prime}, B^{\prime}\right)$ is hereditary and so $I C \cap N=I N \subseteq K N$. Thus it follows that $x \in K N$ and, by Theorem 2.1, that $M$ is E-flat.

COROLLARY 2.5. If $\left(A^{\prime}, B^{\prime}\right)$ is hereditary, then:

(1) every module in $A^{\prime}$ is E-flat;

(2) if $M \in B^{\prime}$, then $M$ is flat if and only if $M$ is E-flat;

(3) every $M \in R^{M}$ is E-flat if and only if $R / I$ is a lvon Neumann) regular ring.

\section{E-flat dimension}

If $M \in R_{R}^{M}$, then we can build a codivisible resolution

$$
\ldots \rightarrow c_{n} \stackrel{\alpha_{n}}{\longrightarrow} \ldots \rightarrow c_{0} \stackrel{\alpha_{0}}{\longrightarrow} M \rightarrow 0
$$

of $M$ where each $C_{i}$ is codivisible with respect to $\left(A^{\prime}, B^{\prime}\right)$ and ker $\alpha_{i} \in B^{\prime}$ for $i \geq 0$. (Notice that $C_{i} \in B^{\prime}$ for $i \geq 1$ since $B^{\prime}$ is closed under extensions.) Indeed, if $\varphi: F \rightarrow M$ is the free module on $M$ and $K=\operatorname{ker} \varphi$, then $F / I K$ is codivisible with respect to $\left(A^{\prime}, B^{\prime}\right)$ and $K / I K \in B^{\prime}$. Thus we need only set $C_{0}=F / I K$ and let $\alpha_{0}$ be the induced mapping. Hence the result follows by induction. Consequently, for any 
$X \in M_{R}$, we have a complex

$$
\ldots \rightarrow X \otimes R_{n}^{C} \rightarrow \ldots \rightarrow X \otimes{ }_{R}^{C} C_{0} \rightarrow
$$

If $\operatorname{tor}_{n}^{E}(X, M)$ denotes the $n$-th homology group of this sequence, then it is easy to show that $\operatorname{tor}_{n}^{E}(X, M)$ is independent of the particular codivisible resolution selected for $M$ and that $\operatorname{tor}_{0}^{E}(X, M) \cong X \otimes{ }_{R}^{M}$. Therefore if $0 \rightarrow L \rightarrow X \rightarrow N \rightarrow 0$ is in $E$, then by tensoring this into (*) and taking homology we obtain an exact sequence

$$
\begin{aligned}
\ldots \rightarrow \operatorname{tor}_{n}^{E}(L, M) & \rightarrow \operatorname{tor}_{n}^{E}(X, M) \rightarrow \operatorname{tor}_{n}^{E}(N, M) \rightarrow \ldots \rightarrow \operatorname{tor}_{1}^{E}(L, M) \\
& \rightarrow \operatorname{tor}_{I}^{E}(X, M) \rightarrow \operatorname{tor}_{I}^{E}(N, M) \rightarrow L \otimes{ }_{R}^{M} \rightarrow X \otimes R_{R}^{M} \rightarrow N \otimes R_{R}^{M} \rightarrow 0 .
\end{aligned}
$$

Thus if $\operatorname{tor}_{n}^{E}(\cdot, M)=0$ for all $n \geq 1$, then $M$ is $E$-flat. To show the converse we need the following lemmas.

LEMMA 3.1. If $\left(A^{\prime}, B^{\prime}\right)$ is hereditary and $0 \rightarrow L \stackrel{f}{\rightarrow} M \stackrel{g}{\rightarrow} N \rightarrow 0$ is exact in $R^{M}$, then the induced sequence $0 \rightarrow L / I L \stackrel{\bar{f}}{\rightarrow} M / I M \stackrel{\bar{g}}{\rightarrow} N / I N \rightarrow 0$ is exact in $R / I^{M}$.

Proof. If $\bar{f}(x+I L)=f(x)+I M=0$, then $f(x) \in I M$. Hence $f(x) \in I M \cap f(L)$. But $\left(A^{\prime}, B^{\prime}\right)$ is hereditary and so $I M \cap f(L)=I f(L)$. If $f(x)=\sum_{i=1}^{n} k_{i} f\left(y_{i}\right) \in I f(L)$, then $x-\sum_{i=1}^{n} k_{i} y_{i} \in$ ker $f=0$. Thus $x \in I L$ and so $\bar{f}$ is a monomorphism. The proofs when im $\bar{f}=\operatorname{ker} \bar{g}$ and $\bar{g}$ is an epimorphism are similar and will therefore be omitted.

LEMMA 3.2. If $\left(A^{\prime}, B^{\prime}\right)$ is hereditary and $O \rightarrow L \rightarrow M \rightarrow N \rightarrow 0$ is exact in $R^{M}$ with $N$ E-flat, then $M$ is E-flat if and only if $L$ is E-flat.

Proof. By the lemma above the induced sequence $0 \rightarrow L / I L \div M / I M \rightarrow N / I N \rightarrow 0$ is exact. Now, by Theorem 2.4, N/IN is a flat $R / I$-module and so, as is well known, $M / I M$ is a flat $R / I$-module if and only if $L / I L$ is a flat $R / I$-module. Hence the result, again by 
Theorem 2.4 .

LEMMA 3.3. If $\left(A^{\prime}, B^{\prime}\right)$ is hereditary and $0 \rightarrow L \rightarrow M \rightarrow N \rightarrow 0$ is exact in $R^{M}$ with $N$ E-flat and $L \in B^{\prime}$, then $0 \rightarrow X \otimes{ }_{R} L \rightarrow X \otimes R_{R}^{M} \rightarrow X \otimes R_{R}^{N \rightarrow 0}$ is exact for any $X \in M_{R}$.

Proof. Consider the commutative diagram

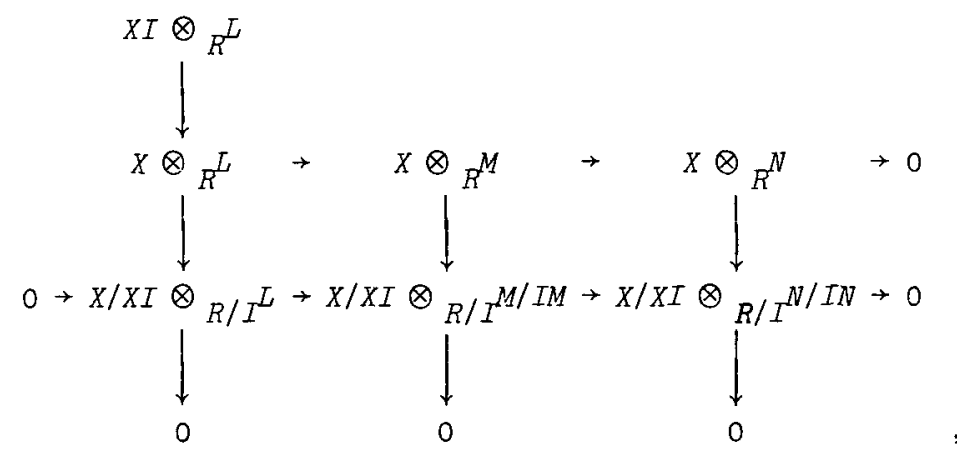

where the columns and rows are exact, the maps being the obvious ones. Notice that the bottom row is exact since $N / I N$ is a flat $R / I$-module. Now $L \in B^{\prime}$ and so $X I \otimes \frac{L}{R}=0$. Hence $X \otimes \otimes_{R} L \rightarrow X / X I \otimes R / I$ is an isomorphism and from this one can see, by chasing around the diagram, that $X \otimes{ }_{R} L \rightarrow X \otimes{ }_{R}^{M}$ is an injection.

Now for the theorem.

THEOREM 3.4. If $\left(A^{\prime}, B^{\prime}\right)$ is hereditary, then $\operatorname{tor}_{n}^{E}(\cdot, M)=0$ for alz $n \geq 1$ if and only if $M$ is E-flat.

Proof. We have already seen that if $\operatorname{tor}_{n}^{E}(\cdot, M)=0$ for all $n \geq 1$, then $M$ is E-flat. Conversely, suppose that $M$ is E-flat and let $\ldots \rightarrow C_{n} \rightarrow \ldots \rightarrow C_{0} \rightarrow M \rightarrow 0$ be a codivisible resolution of $M$ with respect to $\left(A^{\prime}, B^{\prime}\right)$. If $X \in M_{R}$, then by applying Lemma 3.2, Lerma 3.3, and Theorem 2.3, it follows that $\ldots \rightarrow X \otimes{ }_{R}{ }_{n} \rightarrow \ldots \rightarrow X \otimes{ }_{P^{C}} C_{0} \rightarrow X \otimes{ }_{R}{ }^{M} \rightarrow 0$ is exact. Consequently, the result.

Suppose next that $0 \rightarrow L \rightarrow X \rightarrow N \rightarrow 0$ is exact in $R^{M}$ and let $L \in B^{\prime}$; then, by using standard arguments, we can find a short exact 
sequence $0 \rightarrow\left\{C_{n}^{\prime}\right\} \rightarrow\left\{C_{n}\right\} \rightarrow\left\{C_{n}^{\prime \prime}\right\} \rightarrow 0$ of codivisible resolutions of $L, M$, and $N$ respectively. Thus for any $M \in M_{R}$ we have a commutative diagram

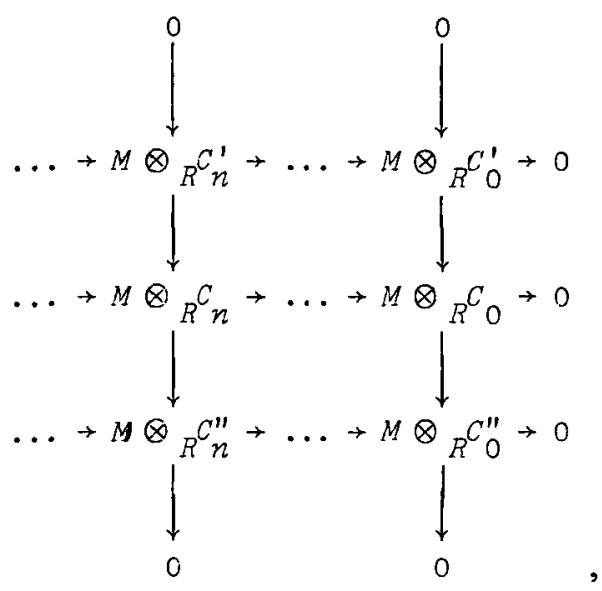

Notice that the columns are exact since each $C_{i}^{\prime \prime}$ is E-flat and $C_{i}^{\prime} \in B^{\prime}$ for $i \geq 0$. Taking homology we obtain an exact sequence

$$
\begin{aligned}
\ldots \rightarrow \operatorname{tar}_{n}^{E}(M, L) & \rightarrow \operatorname{tor}_{n}^{E}(M, X) \rightarrow \operatorname{tar}_{n}^{E}(M, N) \rightarrow \ldots \rightarrow \operatorname{tor}_{1}^{E}(M, L) \\
& \rightarrow \operatorname{tor}_{1}^{E}(M, X) \rightarrow \operatorname{tor}_{1}^{E}(M, N) \rightarrow M \otimes \otimes_{R}^{L} \rightarrow M \otimes \otimes_{R}^{X} \rightarrow M \otimes R_{R}^{N} \rightarrow 0 .
\end{aligned}
$$

From this it follows that $M \otimes{ }_{R}(\cdot)$ preserves short exact sequences $0 \rightarrow L \rightarrow X \rightarrow N \rightarrow 0$ in $R^{M}$ with $L \in B^{\prime}$ if and only if $\operatorname{tor}_{n}^{E}(M, \cdot)=0$ for all $n \geq 1$. Hence we have

THEOREM 3.5. If $\left(A^{\prime}, B^{\prime}\right)$ is hereditary, then the following are equivalent:

(1) $\operatorname{tor}_{n}^{E}=0$ for all $n \geq 1$;

(2) every $M \in{ }_{R}^{M}$ is E-flat;

(3) if $M \in M_{R}$, then $M \otimes_{R}(\cdot)$ preserves short exact sequences $0 \rightarrow L \rightarrow X \rightarrow N \rightarrow 0$ in $R^{M}$ with $L \in B^{\prime}$.

If $M \in{ }_{R} M$, then we define the E-flat dimension of $M$ to be the smallest integer such that $\operatorname{tor}_{n+1}^{E}(\cdot, M)=0$. If $E-f d(M)$ denotes the 
E-flat dimension of $M$, the left global E-flat dimension of $R$ is 1.gl.E-fd $(R)=\sup \left\{E_{-f d}(M) \mid M \in R_{R}^{M\}}\right.$. The following theorem relates the E-flat dimension of $M \in R^{M}$ to the flat dimension of the $R / I$-module $M / I M$. Let $f d(M / I M)$ denote the flat dimension of $M / I M$ as an $R / I-$ module.

THEOREM 3.6. If $\left(A^{\prime}, B^{\prime}\right)$ is hereditary, then for any $M \in R^{M}$, $E-f d(M)=f d(M / I M)$.

Proof. If $\ldots \rightarrow c_{n} \stackrel{\alpha_{n}}{\longrightarrow} \ldots \rightarrow c_{1} \stackrel{\alpha_{1}}{\longrightarrow} c_{0} \stackrel{\alpha_{0}}{\longrightarrow} M \rightarrow 0$ is a codivisible resolution of $M$ with respect to $\left(A^{\prime}, B^{\prime}\right)$, then the induced sequence

$$
\ldots+c_{n} \stackrel{\bar{\alpha}_{n}}{\longrightarrow} \ldots C_{1} \stackrel{\bar{\alpha}_{1}}{\longrightarrow} C_{0} / I C_{0} \stackrel{\bar{\alpha}_{0}}{\longrightarrow} M / I M \rightarrow 0,
$$

where $\bar{\alpha}_{i}=\alpha_{i}$ for $i \geq 2$ is an $R / I$-projective resolution of $M / I M \quad[6$, Theorem 8]. Hence if $E-f d(M)=k$, then $k$ is the smallest integer such that $\operatorname{tor}_{k+1}^{E}(\cdot, M)=0$. Now it is easy to show that

$\operatorname{tor}_{k+1}^{E}(X, M) \cong \operatorname{tor}_{1}^{E}\left(X\right.$, im $\left.\alpha_{k}\right)$ for any $X \in M_{R}$ and so $k$ is the smallest integer such that im $\alpha_{k}$ is E-flat. But $\left(A^{\prime}, B^{\prime}\right)$ is hereditary and therefore im $\alpha_{k} \cap I C_{k-1}=I$ im $\alpha_{k}$. Hence

$$
\text { im } \bar{\alpha}_{k}=\left(\text { im } \alpha_{k}+I C_{k-1}\right) / I C_{k-1} \cong \operatorname{im} \alpha_{k} /\left(\operatorname{im} \alpha_{k} \cap I C_{k-1}\right)=\operatorname{im} \alpha_{k} / I \text { im } \alpha_{k}
$$

and so, by Theorem 2.4, im $\bar{\alpha}_{k}$ is a flat $R / I$-module. In fact, one can show that $k$ is the smallest integer such that im $\bar{\alpha}_{k}$ is a flat

$R / I$-module. Therefore $E-f a(M)=f a(M / I M)$.

COROLLARY 3.7. If $\left(A^{\prime}, B^{\prime}\right)$ is hereditary, then l.gl.E-fd $(R)=1 . g l \cdot f d(R / I)$.

In [6], Rangaswamy has defined a codivisible dimension for modules in $R^{M}$ and a left global codivisible dimension for $R$ with respect to any hereditary torsion $\left(A^{\prime}, B^{\prime}\right)$ on $R^{M}$. Briefly, if 
$\ldots \rightarrow c_{n} \stackrel{\alpha_{n}}{\longrightarrow} \ldots \rightarrow c_{0} \stackrel{\alpha_{0}}{\longrightarrow} M \rightarrow 0$ is a codivisible resolution of $M$ with respect to $\left(A^{\prime}, B^{\prime}\right)$, then the codivisible dimension of $M$ is the smallest integer $n$ such that im $\alpha_{n}$ is codivisible. The I.gl.cod $(R)$ (the left global codivisible dimension of $R$ ) is then defined in the obvious way. Rangaswamy has shown [6, Theorem 14] that the left global codivisible dimension of $R$ equals the left global homological dimension of $R / A^{\prime}(R)$ where $A^{\prime}(R)$ is the torsion ideal of $R$ with respect to $\left(A^{\prime}, B^{\prime}\right)$. If $E^{\prime}$ is the class of all short exact sequences $0 \rightarrow L \rightarrow X \rightarrow N \rightarrow 0$ in $R^{M}$ with $N \in B^{\prime}$ and $\left(A^{\prime}, B^{\prime}\right)$ and $(A, B)$ are both hereditary, when viewing the obvious symmetry of our work we see that l.gl.E-fd $(R)=\mathrm{r} . \mathrm{gl} . E-f d(R)$. Since the left global flat dimension of a left noetherian ring coincides with its left global homological dimension, we conclude with the following observation.

THEOREM 3.8. If $\left(A^{\prime}, B^{\prime}\right)$ is hereditary and the left ideals in $F^{\prime}(R)=\{K \mid K \supseteq I, K$ a left ideal of $R\}$ satisfy the ascending chain condition, then l.gl.E-fd $(R)=1 . \mathrm{gl} \cdot \operatorname{cod}(R)$.

\section{References}

[1] P.E. Bland, "Perfect torsion theories", Proc. Amer. Math. Soc. 41 (1973), 349-355.

[2] Spencer E. Dickson, "A torsion theory for abelian categories", Trans. Amer. Math. Soc. 121 (1966), 223-235.

[3] Oscar Goldman, "Rings and modules of quotients", J. Algebra 13 (1969), $10-47$.

[4] J.P. Jans, "Some aspects of torsion", Pacific J. Math. 15 (1965), 1249-1259.

[5] Joachim Lambek, Torsion theories, additive semantics and rings of quotients (Lecture Notes in Mathematics, 177. Springer-Verlag, Berlin, Heidelberg, New York, 1971).

[6] K.M. Rangaswamy, "Codivisible modules", Comm. Algebra 2 (1974), $475-489$. 
[7] Bo Stenström, Rings and modules of quotients (Lecture Notes in Mathematics, 237. Springer-Verlag, Berlin, Heidelberg, New York, 1971).

Department of Mathematics,

Eastern Kentucky University,

Richmond,

Kentucky,

USA. 